\title{
An active/passive joint for reconfiguration applications
}

\author{
M. Riabtsev, V. Petuya, A. Riera and M. Urízar \\ Department of Mechanical Engineering. University of the Basque Country UPV/EHU \\ Plaza Torres Quevedo 1, 48013 Bilbao, Spain \\ mriabtsev001@ikasle.ehu.eus
}

\begin{abstract}
In this paper, an active/passive reconfigurable $2 \mathrm{R}$ joint is presented. Direct and inverse kinematic problems and the force transmission are considered. The advantages of the current design show its superiority with respect to previous designs. A prototype of the joint was built and tested, proving the viability of the concept. The proposed joint can be applied in reconfigurable manipulators, serial robot arm wrists, humanoid robot shoulder and waist, where $2 \mathrm{R}$ active joints are required.
\end{abstract}

Keywords: active/passive joint, manipulator, reconfigurable, prototype.

\section{Introduction}

The process of readjusting an assembly line for a new product is very costly if it involves equipment replacement. One way of reducing cost is to use reconfigurable mechanisms, which can be readjusted for a new task and do not require complete replacement.

There are different reconfiguration strategies. The example of the offline reconfiguration approach is modular reconfiguration [1], when mechanisms are made from standard modules which can be easily reconnected in the required way. Other methods lay on additional actuation [2], or on the contrary, locking degrees of freedom [3]. Some research is focused on the reconfiguration by changing the dimensions of the base or the end-effector platforms [4] or other links of the mechanism [5]. Another widely used way of changing the mechanism's topology is the application of reconfigurable joints. These joints can combine different types of motion [6], change orientation of their axis [7] or involve other strategies [8-10].

In the current paper, a $2 \mathrm{R}$ active/passive reconfigurable joint is presented. The design of the joint is described in Section 2, kinematics and force transmission analysis is provided in Section 3, prototype manufacturing is described in Section 4. Finally, possible applications and future work are highlighted.

\section{Design description}

The joint proposed in this paper (Fig. 1) consists of a central ball with a slot crossing the sphere. The red input is placed in this slot and defines the first rotational input. The 
blue shaft transmits rotation to the ball through the bevel gear to control the second rotational degree of freedom. If two inputs are used (Fig. 1) in order to increase the accuracy and balance the joint, the input command for the two light blue shafts should have the same value but in opposite directions.

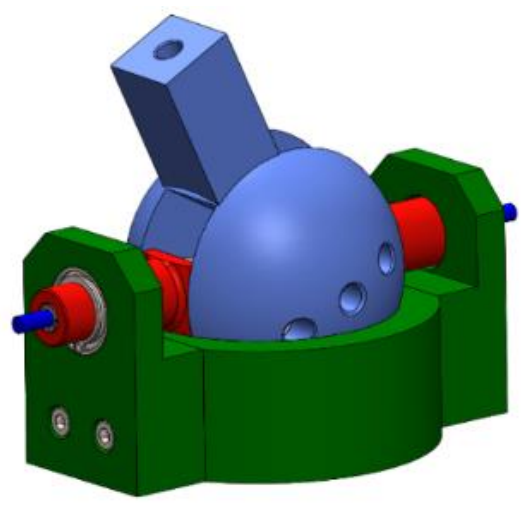

a)

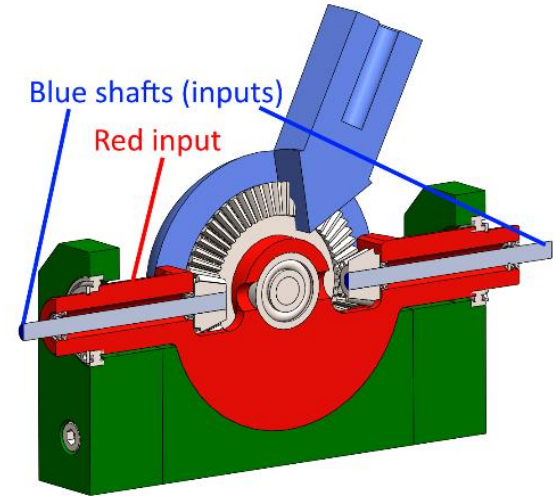

b)

Fig. 1. CAD model of the proposed joint: a) general view; b) cross section.

The proposed joint is kinematically a cardan joint with the capacity to be actuated in both axes. The design proposed in this paper, aims at increasing the load carrying capacity of the joint compared to the conventional universal joints. Thus, the contact area between the central ball and the base part allows a good distribution of the load.

The proposed joint represents an evolution of a previous design shown in Fig. 2 [11], which was based in the concept of the spherical five bar linkage. This previous design had several drawbacks. Firstly, the relation between the input commands in the blue shafts depends on the input command in the red one. In addition, the clearances between the sliding parts are hard to compensate and the value of transmitted torque depends on the dimensional parameters of the joint (ball radius, link lengths). Finally, the structural elements of the joint make workspace extension impossible.

In the proposed joint, most of these drawbacks have been eliminated. The workspace volume is now $73.9 \%$ bigger due to the absence of the top ring and elimination of the singularity position inherent to the previous design. The joint has increased the percentage of the ball-to-base contact (from $60.87 \%$ to $93.22 \%$ ) because of the open slots elimination. The actuation is now uniform and symmetrical. The use of two actuators for the blue shafts creates a pretension in the gear that eliminates the clearances and improves the accuracy of the joint. In addition, the kinematics of the joint are very simple and there is the possibility of one-side actuation that can be useful for some applications. 


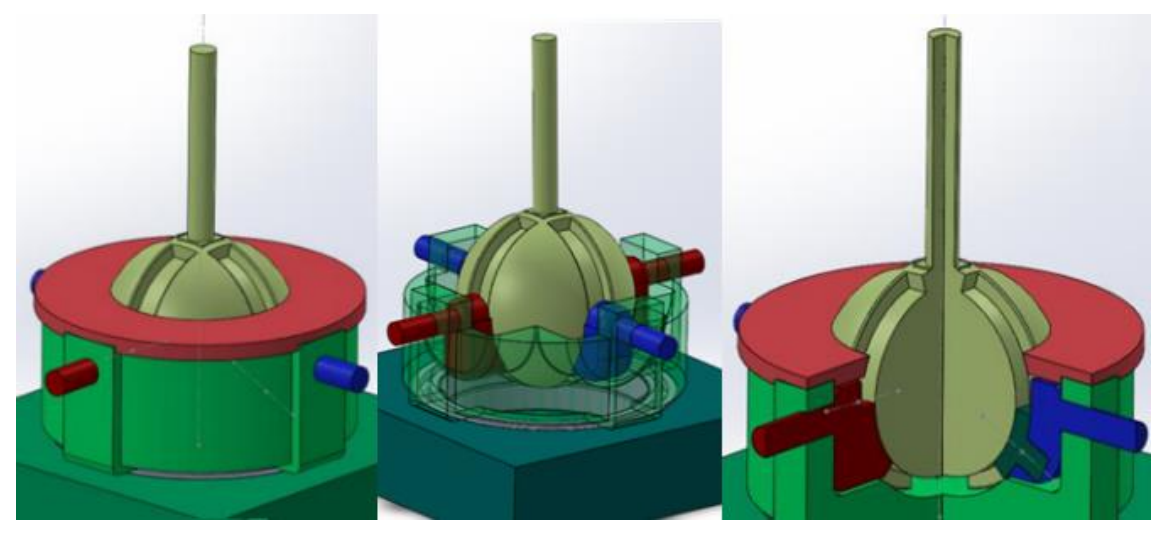

Fig. 2. Initial design [11]

Between the initial design shown in Fig. 2 and the presented design shown in Fig. 3, other alternatives have been proposed. For example, in the joint of Fig. 3 the main design goals were the kinematics simplification and the increase of the workspace volume. In this design, the workspace volume is $236,4 \%$ bigger in comparison to the design shown on Fig. 2, due to the actuators arrangement and the elimination of the elements, which can limit the joint movement. Unfortunately, this solution implied the elimination of the joint ball, thus affecting the load capacity. The other disadvantage of this design is the clearance in the gear, which is hard to compensate and affects the accuracy of the joint movement.

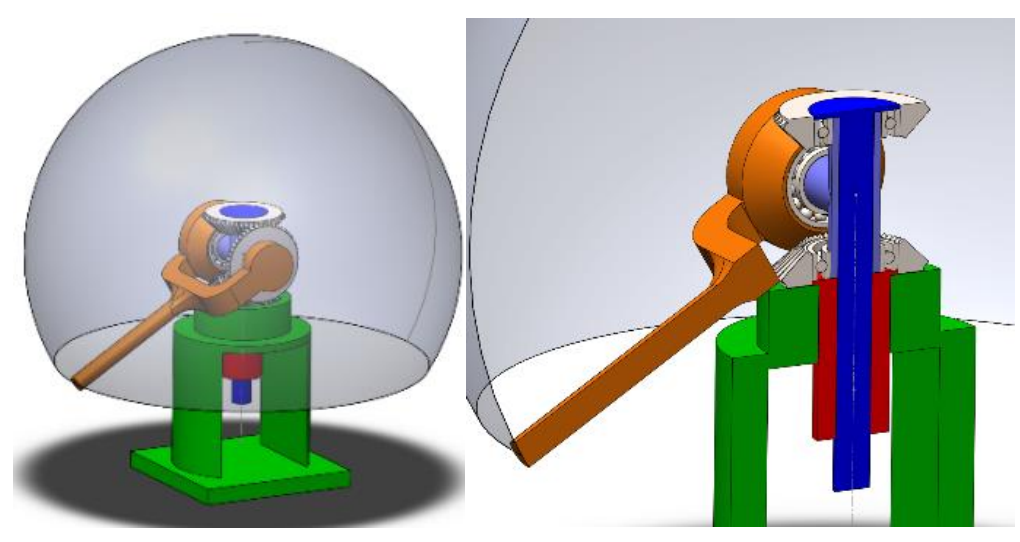

Fig. 3. Intermediate design: joint with extended workspace

As explained, the new joint can be simultaneously actuated to control the two rotational degrees of freedom. Additionally, the joint can be implemented in a reconfigurable manipulator as an active/passive rR joint. In this case, the red input defines de orientation of the rotation axis and once it is blocked, a reconfigurable revolute joint is obtained. 


\section{$3 \quad$ Kinematic analysis of the joint}

\subsection{Direct position problem}

Consider the joint with input angles $\alpha$ for the red shaft and $\beta_{\text {input }}$ for the blue shaft. After an input of $\alpha$ in the red shaft, the vector defining the orientation of the central rod (Fig. 4) is,

$$
n^{\prime}=\left\{\begin{array}{c}
0 \\
-\sin \alpha \\
\cos \alpha
\end{array}\right\}
$$

The input $\beta_{\text {input }}$ causes a rotation of $\beta$ around the $V^{\prime}$ axis being,

$$
\beta=\left(\frac{z_{1}}{z_{2}}\right) \beta_{\text {input }} \pm \alpha
$$

where $Z_{1}$ and $Z_{2}$ are the number of teeth in the pinion and in the gear.

Thus, the orientation vector of the central rod is calculated as follows,

$$
\mathrm{n}=\left[\begin{array}{ccc}
\cos \beta & 0 & \sin \beta \\
0 & 1 & 1 \\
-\sin \beta & 0 & \cos \beta
\end{array}\right]\left\{\begin{array}{c}
0 \\
-\sin \alpha \\
\cos \alpha
\end{array}\right\}=\left\{\begin{array}{c}
\sin \beta \cos \alpha \\
-\sin \alpha \\
\cos \beta \cos \alpha
\end{array}\right\}
$$

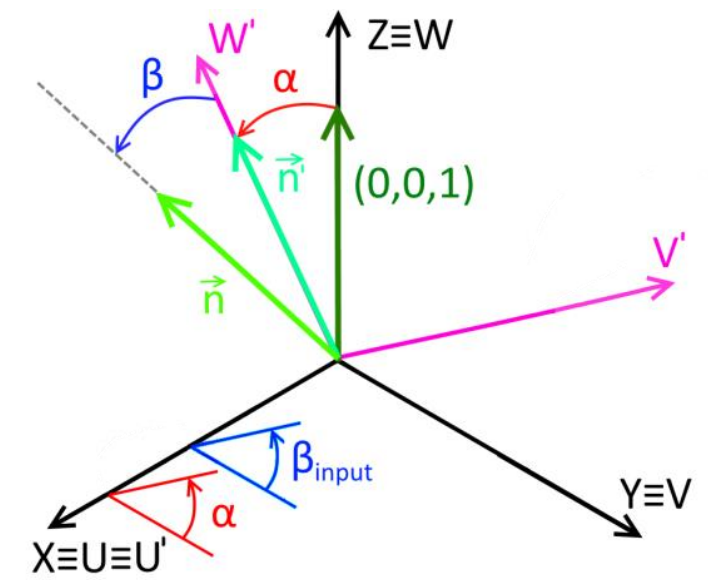

Fig. 4. Position parameters of the joint. 
which normalized, has the form,

$$
n=\left\{\begin{array}{c}
\tan \beta \\
-\tan \alpha / \cos \beta \\
1
\end{array}\right\}
$$

\subsection{Inverse position problem}

In this case, the components of vector $n=\left\{\begin{array}{lll}u & v & 1\end{array}\right\}^{T}$ are known. Thus, the values for the red and blue inputs can be directly calculated as,

$$
\begin{gathered}
\beta=\tan ^{-1} u \\
\alpha=-\tan ^{-1}(v \cos \beta)
\end{gathered}
$$

being (2) the relation between $\beta$ and $\beta_{\text {input }}$.

\subsection{Force transmission in the joint}

As it is shown subsequently, the computation of the force transmission becomes rather simple for the proposed joint. As the joint has two inputs, two input torques should be considered. Red input torque $T_{r}$ creates red force component $F_{r}$ :

$$
\mathrm{F}_{\mathrm{r}}=\frac{\mathrm{T}_{\mathrm{r}}}{\mathrm{d}_{\mathrm{r}}}
$$

where $d_{r}$ is the shortest distance between the point where the force $F_{r}$ is applied and the axis of rotation of red input (

Fig. 5). This distance is calculated as,

$$
\mathrm{d}_{\mathrm{r}}=\mathrm{R} \cos \beta
$$

being, $R$ the distance between the application point and the center of the joint ball. The direction of the force $F_{r}$ is $\left(-V^{\prime}\right)$.

Additionally, blue input torque $T_{b}$ creates red force component $F_{b}$ :

$$
F_{b}=\left(\frac{z_{2}}{z_{1}}\right) T_{b} R
$$


This feature provides additional force amplification in the joint and can be considered as an advantage for certain applications. The direction of the force $F_{r}$ is on plane $W^{\prime} U^{\prime}$ and perpendicular to vector $\boldsymbol{n}$ (Fig. 4).

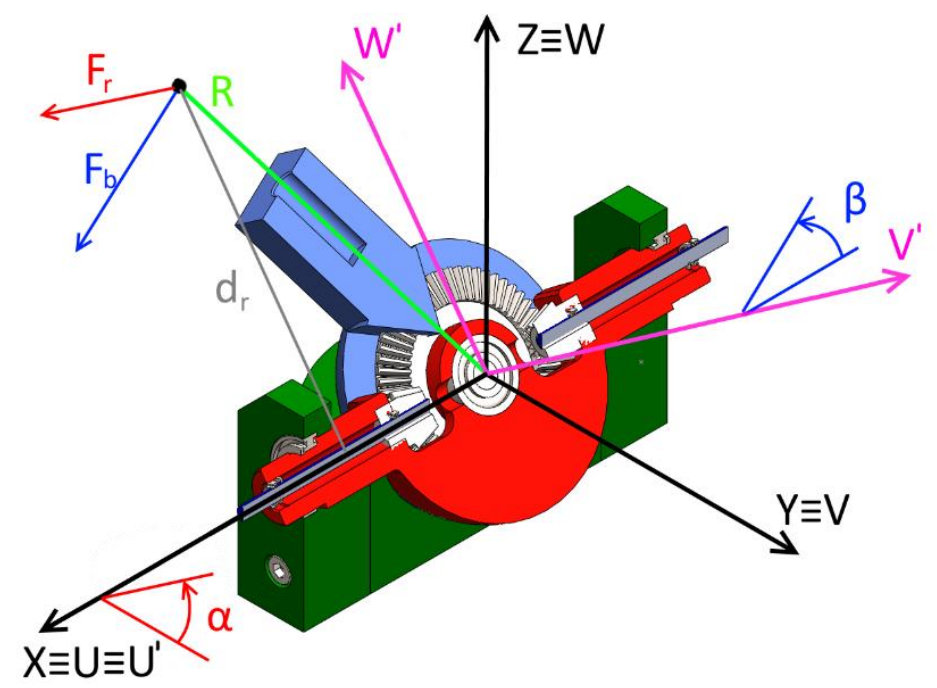

Fig. 5. Force transmission in the joint

The two force components are perpendicular to each other, thus the module of the resultant force yields:

$$
\mathrm{F}=\sqrt{\mathrm{F}_{\mathrm{r}}^{2}+\mathrm{F}_{\mathrm{b}}^{2}}
$$

\section{$4 \quad$ Prototype manufacturing}

For testing the proposed joint, a demonstrative prototype was manufactured (Fig. 6). The base of joint, the ball and the red input were printed with Stratasys' Dimension sst1200. The bevel gear and the pinions were printed with Markforged Mark Two 3D printer, which provides better surface quality.

The mechanical properties of the printed gears and their accuracy do not allow implementing this prototype directly in a manipulator, but, overall, it becomes an effective way for analyzing the concept of the proposed joint.

The testing of the prototype demonstrated the lack of durability of the gear teeth. Currently, the design of the joint is being improved with the aim of building a practical joint using steel/aluminum, which will be able to be used in a reconfigurable mechanism. This process requires modification of each part of the joint for the conventional 
machining methods, because 3D printing cannot provide satisfactory tolerances and the surface quality.

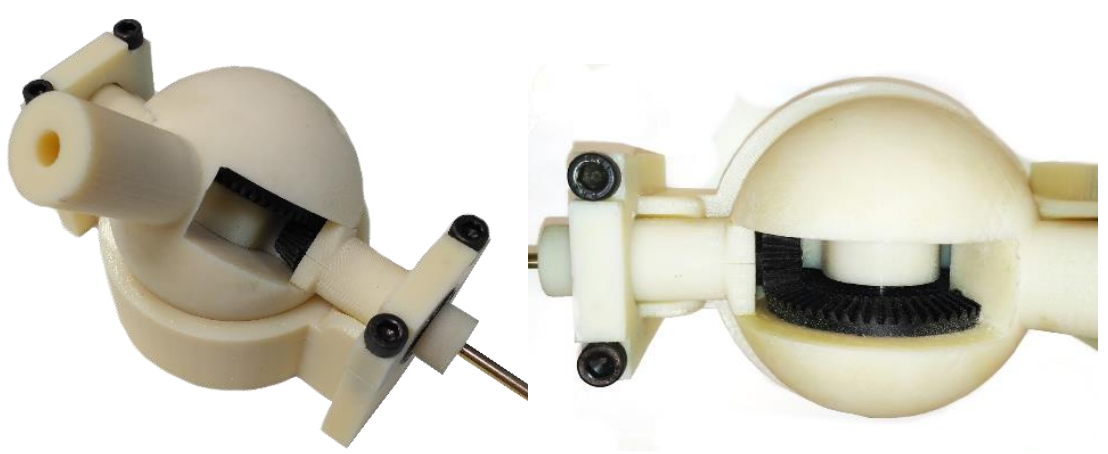

Fig. 6. Demonstrative prototype

Among the possible applications, the joint presented in this paper can be incorporated in parallel robots, act as a wrist of a serial robotic arm, shoulder joint or waist of a humanoid robot, walking robot leg joints, etc.

\section{Conclusions}

In this paper, a 2R active/passive joint has been presented. The proposed joint is an evolution of previous designs and it demonstrates improved workspace, simpler input control and higher load carrying capacity and accuracy. In addition, there is the possibility of one-side actuation that can be useful for some applications. The joint can be applied as a reconfigurable $\mathrm{rR}$ joint which is able to change the orientation of its axis, or as a lockable universal joint.

Currently we are working on the design improvement in order to build a prototype in steel/aluminum. In addition, we are working on finite element structural analysis of the joint to find out the weaknesses and limitations of the current design.

\section{Acknowledgments}

The authors wish to acknowledge the financial support received from the Spanish Government through the Ministerio de Economía y Competitividad (Project DPI2015-67626-P (MINECO/FEDER, UE)) and the financial support given to the research group through the project with Ref. IT949-16, given by the Departamento de Educación, Política Lingüística y Cultura of the Regional Government of the Basque Country.

\section{References}

1. I.-M. Chen, "Rapid response manufacturing through a rapidly reconfigurable robotic workcell," Robotics and Computer Integrated Manufacturing, 17, pp. 199-213, 2001. 
2. K. Wohlhart, (1996) Kinematotropic Linkages. In: Lenarčič J., Parenti-Castelli V. (eds) Recent Advances in Robot Kinematics, pp. 359-368. Springer, Dordrecht

3. P. Grosch, R. Di Gregorio, J. López and F. Thomas, "Motion planning for a novel reconfigurable parallel manipulator with lockable revolute joints," In Proceedings of the IEEE 2010 International Conference on Robotics and Automation (ICRA), Anchorage, AK, USA, 3-7 May 2010, pp. 4697-4702.

4. C. Viegas, M. Tavakoli and Aníbal T. de Almeida, "A novel grid-based reconfigurable spatial parallel mechanism with large workspace," Mechanism and Machine Theory, vol. 115, pp. 149-167, 2017.

5. S. Nansai, N. Rojas, M. Elara and R. Sosa, "Exploration of adaptive gait patterns with a reconfigurable linkage mechanism," In Proceedings of the 2013 IEEE/RSJ International Conference on Intelligent Robots and Systems (IROS 2013), Tokyo, 2013, pp. 4661-4668.

6. H. S. Yan and C. H. Kuo, "Topological Representations and Characteristics of Variable Kinematic Joints," Journal of Mechanical Design, vol. 128, pp. 384-391, March 2006.

7. D. Gan, J. S. Dai and Q. Liao, "Mobility Change in Two Types of Metamorphic Parallel Mechanisms," Journal of Mechanisms and Robotics, vol. 1, pp. 041007-1-041007-9, 2009.

8. Y. Wei, Y. Fang and S. Guo, "Reconfigurable Parallel Mechanisms with Three Types of Kinematotropic Chains," In Proceedings of the $14^{\text {th }}$ International Federation for the Promotion of Mechanism and Machine Science (IFToMM) World Congress, Taipei, Taiwan, 2530 October, 2015.

9. P. A. Halverson, L. Howell and S. Magleby, "Tension-based multi-stable compliant rollingcontact elements," Mechanism and Machine Theory, vol. 45, Issue 2, pp. 147-156, 2010.

10. C. Lee and J. Hervé, "Various types of RC//RC -like linkages and the discontinuously movable Koenigs joint," Mechanism and Machine Theory, vol. 96, pp. 255-268, 2015.

11. M. Riabtsev, V. Petuya, A. Riera and E. Macho, " Design of an active reconfigurable 2R joint," In Proceedings of the 15th International Federation for the Promotion of Mechanism and Machine Science (IFToMM) World Congress, Krakov, 30-4 July, 2019. 